\section{Performance of Delay and Add Direct Sequence Spread Spectrum Modulation Scheme with Fast Frequency Hopping in Frequency Selective Rayleigh Channels}

Vincent Le Nir, Bart Scheers

\begin{abstract}
The coherent direct-sequence spread spectrum (DSSS) modulation is a very attractive modulation technique for military communication systems, mainly due to its resistance to interference and low probability of detection (LPD). However, the synchronisation to the spreading code phase in the receiver takes time, which makes this technique less interesting for sending very short messages or to combine this technique with frequency hopping. Recently, a new non-coherent DSSS modulation scheme, called the delay and add direct sequence (DADS) modulation scheme, based on the transmit reference (TR) principle has been introduced. DADS has demonstrated to be particularly suited for short burst transmissions and can be combined with a fast frequency hopping scheme. In this paper, the performance of the DADS modulation scheme in combination with fast frequency hopping in frequency selective Rayleigh channels is studied. Theoretical analysis and simulation results show that the BER performance of DADS is improved drastically in frequency selective Rayleigh channels although keeping the same noncoherent receiver. Moreover, the BER performance of DADS is also enhanced with fast frequency hopping due to an increase in the diversity order of the frequency selective Rayleigh channels and provides LPD/LPI to the modulation scheme.
\end{abstract}

Index Terms-Spread spectrum modulation scheme, transmit reference, non-coherent detection

\section{INTRODUCTION}

The direct-sequence spread spectrum (DSSS) modulation is a very attractive modulation technique for military communication systems, mainly due to its resistance to interference and low probability of detection (LPD). The main drawback of DSSS lies in the complexity of the receiver. Indeed, along with the usual carrier tracking as in a traditional digital receiver, a code alignment is needed for a correct despreading. The carrier and code tracking are respectively done by a phaselocked loop (PLL) and a delay-locked loop (DLL). The DLL is normally based on an early-late-prompt sampler. For the PLL, a Costas loop can be used. The particularity of the two loops however is that they are entwined, meaning that the output of one loop serves as input of the other and vice-versa. A second drawback, which is directly connected to the first, is the time needed to synchronise on the spreading code. In fact, to bring the DLL in its domain of operation, a first rough code phase estimation is needed. This coarse estimation is performed by an acquisition block, and is based on the evaluation of an ambiguity function. As a consequence, a long preamble is advisable, which makes the DSSS modulation less suitable for

V. Le Nir and B. Scheers are with the Royal Military Academy, Dept. Communication, Information Systems \& Sensors (CISS), 30, Avenue de la Renaissance B-1000 Brussels BELGIUM. E-mail: bart.scheers@rma.ac.be vincent.lenir@rma.ac.be

This research work was carried out in the frame of the Belgian Defense Scientific Research \& Technology Study C4/19 funded by the Ministry of Defense (MoD). The scientific responsibility is assumed by its authors. transmission of short messages or to combine this technique with frequency hopping.

Correlation delay shift keying (CDSK) has been introduced by Sushchik in 2000 [1] and extended to the generalized CDSK (GCDSK) by Tam in 2006 [2]. CDSK or GCDSK is a transmit reference (TR) modulation scheme primarily designed for non-coherent detection in which a chaotic signal is used on one hand as an embedded reference signal, and on the other hand for modulating the data information. This modulation scheme provides a processing gain and therefore inherits the advantage of conventional spread-spectrum communications such as LPD, multipath mitigation, anti-jamming and multiple access capabilities. Moreover, contrary to coherent detection, non-coherent detection does not require the phase synchronisation of the spreading code at the receiver, resulting in a very simple receiver structure.

Recently, a new modulation scheme utilising pseudorandom binary sequences (PRBS) instead of chaotic reference signals has been defined as the delay and add direct sequence (DADS) modulation scheme [3]. The DADS modulation scheme has lower complexity than the DSSS modulation scheme and is particularly suited for short burst transmissions. The transmitted signal is composed of an DSSS modulated signal added together with a delayed version of the spreading code, as in traditional TR methods such as CDSK. This delayed version is exploited for the despreading in the noncoherent receiver. In this way, there is no more need for code and carrier synchronisation at the receiver side, which makes it possible to combine the DADS modulation efficiently with a fast frequency hopping scheme. As an additional advantage, a fast frequency hopping scheme will reduce the probability of interception (LPI).

The performance of the DADS modulation scheme has been studied in additive white Gaussian noise (AWGN) and flat Rayleigh channels [3], [4]. In this paper, the performance of the DADS modulation scheme is studied with fast frequency hopping in frequency selective Rayleigh channels. The remainder of this paper is organised as follows. In Section II, a theoretical analysis of the BER performance of the DADS modulation scheme in frequency selective Rayleigh channels is presented. In section III, a theoretical analysis of the BER performance of the DADS modulation scheme with fast frequency hopping in frequency selective Rayleigh channels is presented. In section IV, simulation results are given with and without fast frequency hopping in frequency selective Rayleigh channels.

\section{TheOretiCAl ANALYSis OF THE BER PERFoRMANCE OF THE DADS MODULATION SCHEME IN FREQUENCY Selective Rayleigh ChanNels}

The DADS transmission chain is shown on Figure 1. The transmitted signal is the sum of two signals, namely a reference signal and its delayed version multiplied by the information signal. Then it passes through a propagation channel. At the receiver side, the signal is correlated with its delayed conjugate version. Let us derive the mathematics behind Figure 1. We consider a frequency selective Rayleigh 


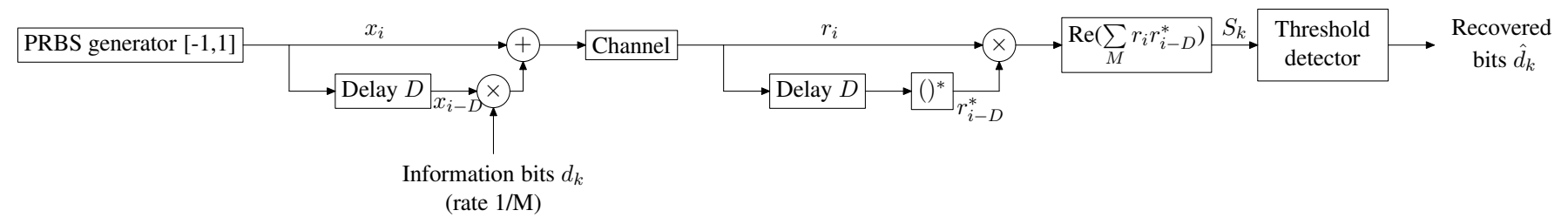

Fig. 1. Transmission chain of the DADS modulation scheme

channel with additive white Gaussian noise (AWGN). The received signal $\left\{r_{i}\right\}$ can be modeled as

$$
r_{i}=\sum_{l=0}^{L-1} h_{l}\left(d_{k} x_{i-l-D}+x_{i-l}\right)+n_{i}
$$

with $L$ the number of taps, $D$ the delay (in chips), $h_{l}$ the complex-valued channel attenuation for the $l^{\text {th }}$ tap, $d_{k}$ the information bits taking values in $\{-1,1\}$ with data rate $1 / M, x_{i}$ the generated bipolar PRBS of length $M$ and $n_{i}$ the AWGN with variance $N_{0} / 2$ per dimension. The correlator output is given by

$$
S_{k}=\operatorname{Re}\left(\sum_{i=(k-1) M+1}^{k M} r_{i} r_{i-D}^{*}\right),
$$

with

$$
\begin{aligned}
r_{i} r_{i-D}^{*}= & \underbrace{d_{k} \sum_{l=0}^{L-1}\left|h_{l}\right|^{2} x_{i-l-D}^{2}}_{\text {useful part } a_{i}} \\
& +d_{k} \sum_{l=0}^{L-1}\left|h_{l}\right|^{2} x_{i-l} x_{i-l-2 D} \\
& +\sum_{l=0}^{L-1}\left|h_{l}\right|^{2} x_{i-l} x_{i-l-D}+\sum_{l=0}^{L-1}\left|h_{l}\right|^{2} x_{i-l-D} x_{i-l-2 D} \\
& \underbrace{+\sum_{l=0}^{L-1} \sum_{l^{\prime} \neq l} h_{l} h_{l^{\prime}}^{*}\left(d_{k} x_{i-l-D}+x_{i-l}\right)\left(d_{k} x_{i-l^{\prime}-2 D}+x_{i-l^{\prime}-D}\right)}_{\text {interference part } b_{i}} \\
& \underbrace{+\sum_{l=0}^{L-1} h_{l} n_{i-D}^{*}\left(d_{k} x_{i-l-D}+x_{i-l}\right)}_{\text {noise part } c_{i}}
\end{aligned}
$$

The first line of the equation corresponds to the useful part $a_{i}$. The second, third and fourth lines corresponds to the interference part $b_{i}$, which has $3 L+4 L(L-1)$ terms due to the cross-correlation between two delayed time intervals of the reference signal. The remaining lines correspond to the noise part $c_{i}$, which has $4 L+1$ terms due to the crosscorrelation between the reference signal and the noise (the last component being the cross-correlation of the noise with its delayed version).

Let us define the output of the correlator corresponding to the useful part $A_{k}$, interference part $B_{k}$ and noise part $C_{k}$

$$
\left\{A_{k}, B_{k}, C_{k}\right\}=\operatorname{Re}\left(\sum_{i=(k-1) M+1}^{k M}\left\{a_{i}, b_{i}, c_{i}\right\}\right) .
$$

\section{A. Arbitrary Pseudo Random Binary Sequence for DADS}

Considering an arbitrary PRBS for the DADS modulation scheme and assuming that the delay $D<\tau_{\max }$, some of the components in (3) become correlated. This motivates the choice of $D$ larger than the maximum delay spread $\tau_{\max }$ (in chips), but significantly lower than the length of the spreading code $M\left(\tau_{\max } \leq D \ll M\right)$. Because $x_{i}$ is statistically independent from $x_{j}$ for any $i \neq j, x_{i}$ is statistically independent from $n_{j}$ for any $(i, j)$, and $n_{i}$ is statistically independent from $n_{j}$ for any $i \neq j$, the useful part $A_{k}$, the interference part $B_{k}$ and the noise part $C_{k}$ can be approximated as Gaussian variables as $M$ increases having the following means

$$
\begin{aligned}
E\left[A_{k}\right] & =d_{k} \sum_{l=0}^{L-1}\left|h_{l}\right|^{2} M P_{s} \\
E\left[B_{k}\right] & =0 \\
E\left[C_{k}\right] & =0
\end{aligned}
$$

with $P_{s}$ the energy per chip and variances

$$
\begin{aligned}
\operatorname{var}\left[A_{k}\right] & =0 \\
\operatorname{var}\left[B_{k}\right] & =3 \sum_{l=0}^{L-1}\left|h_{l}\right|^{4} M P_{s}^{2} \\
& +4 \sum_{l=0}^{L-1} \sum_{l^{\prime} \neq l}\left|h_{l}\right|^{2}\left|h_{l^{\prime}}\right|^{2} M P_{s}^{2} \\
\operatorname{var}\left[C_{k}\right] & =4 \sum_{l=0}^{L-1}\left|h_{l}\right|^{2} M P_{s} \frac{N_{0}}{2}+M \frac{N_{0}^{2}}{2}
\end{aligned}
$$

A semi-analytical approach to evaluate the bit error rate (BER) performance is to average the conditional BER over the channel realisations $h_{l}$ [5]. The BER formula [6] is then given by

$$
\begin{aligned}
B E R & =\frac{1}{2} E_{h_{l}}\left[\operatorname{Prob}\left(S_{k}<0 \mid d_{k}=+1\right)\right. \\
& \left.+\operatorname{Prob}\left(S_{k} \geq 0 \mid d_{k}=-1\right)\right] \\
& =\frac{1}{4} E_{h_{l}}\left[\operatorname{erfc}\left(\frac{E\left[S_{k} \mid d_{k}=+1\right]}{\sqrt{2\left(\operatorname{var}\left[S_{k} \mid d_{k}=+1\right]\right)}}\right)\right. \\
& \left.+\operatorname{erfc}\left(\frac{-E\left[S_{k} \mid d_{k}=-1\right]}{\sqrt{2\left(\operatorname{var}\left[S_{k} \mid d_{k}=-1\right]\right)}}\right)\right]
\end{aligned}
$$

with $E_{h_{l}}$ l.] the expected value over the channel realisations and $\operatorname{erfc}($.$) the complementary error function. Knowing that$ a transmitted data bit is the sum of two sequences of length $M$, the energy per bit $E_{b}$ can be written as $E_{b}=2 M P_{s}$, 
the derivation of the BER formula leads to the following expression

$$
B E R=\frac{1}{2} E_{h_{l}}\left[\operatorname{erfc}\left(\sqrt{\frac{\sum_{l=0}^{L-1}\left|h_{l}\right|^{2} E_{b}}{8 N_{0} \Gamma}}\right)\right]
$$

with

$$
\Gamma=1+\frac{3 \sum_{l=0}^{L-1}\left|h_{l}\right|^{4} E_{b}}{4 \sum_{l=0}^{L-1}\left|h_{l}\right|^{2} M N_{0}}+\frac{M N_{0}}{2 \sum_{l=0}^{L-1}\left|h_{l}\right|^{2} E_{b}}+\frac{\sum_{l=0}^{L-1} \sum_{l^{\prime} \neq l}\left|h_{l}\right|^{2}\left|h_{l^{\prime}}\right|^{2} E_{b}}{\sum_{l=0}^{L-1}\left|h_{l}\right|^{2} M N_{0}}
$$

In the case of a flat Rayleigh channel $(L=1),(8)$ and (9) reduces to

$$
B E R=\frac{1}{2} E_{h_{l}}\left[\operatorname{erfc}\left(\sqrt{\frac{\left|h_{l}\right|^{2} E_{b}}{8 N_{0}\left(1+\frac{3\left|h_{l}\right|^{2} E_{b}}{4 M N_{0}}+\frac{M N_{0}}{2\left|h_{l}\right|^{2} E_{b}}\right)}}\right)\right]
$$

which is consistent with the formula derived in [4].

\section{B. Pseudo Random Binary Sequence Selection for DADS}

The DADS modulation offers the possibility to select some PRBS which improves the BER performance. Indeed, theoretical analysis and simulation results have shown that the bit error rate (BER) performance of DADS with arbitrary PRBS is comparable to CDSK, however the DADS performance can be improved by $3 \mathrm{~dB}$ with PRBS selection [4]. In this Section, we recall the criterion proposed for PRBS selection. The idea is to select the sequence $\left\{x_{i}\right\}$ such that the first term of the interference part $b_{i}$ in (3) moves to the useful part $a_{i}$ while keeping the same properties of the other components in the interference part. This can be done under the following conditions

$$
\text { select }\left\{x_{i}\right\}=\left\{\begin{array}{l}
x_{i}=x_{i-2 D} \\
E\left[x_{i} x_{i-D}\right]=0
\end{array} \quad \forall i\right.
$$

Using this criterion and considering frequency selective Rayleigh channels, (3) can be re-written as

$$
\begin{aligned}
r_{i} r_{i-D}^{*}= & \underbrace{d_{k} \sum_{l=0}^{L-1}\left|h_{l}\right|^{2}\left(x_{i-l-D}^{2}+x_{i-l}^{2}\right)}_{\text {useful part } a_{i}} \\
& +2 \sum_{l=0}^{L-1}\left|h_{l}\right|^{2} x_{i-l} x_{i-l-D} \\
& \underbrace{+\sum_{l=0}^{L-1} \sum_{l^{\prime} \neq l} h_{l} h_{l^{\prime}}^{*}\left(d_{k} x_{i-l-D}+x_{i-l}\right)\left(d_{k} x_{i-l^{\prime}}+x_{i-l^{\prime}-D}\right)}_{\text {interference part } b_{i}} \\
& +\sum_{\text {noise part } c_{i}}^{L-1} h_{l} n_{i-D}^{*}\left(d_{k} x_{i-l-D}+x_{i-l}\right)
\end{aligned}
$$

Therefore, by selecting a spreading code according to (11), a $3 \mathrm{~dB}$ gain on the useful part is achieved while reducing the interference part by one component. Considering a PRBS for the DADS modulation scheme and assuming that the delay $\tau_{\max } \leq D \ll M$, the useful part $A_{k}$, the interference part $B_{k}$ and the noise part $C_{k}$ can be approximated as Gaussian variables as $M$ increases with means

$$
\begin{aligned}
E\left[A_{k}\right] & =2 d_{k} \sum_{l=0}^{L-1}\left|h_{l}\right|^{2} M P_{s} \\
E\left[B_{k}\right] & =0 \\
E\left[C_{k}\right] & =0
\end{aligned}
$$

and variances

$$
\begin{aligned}
\operatorname{var}\left[A_{k}\right] & =0 \\
\operatorname{var}\left[B_{k}\right] & =4 \sum_{l=0}^{L-1}\left|h_{l}\right|^{4} M P_{s}^{2} \\
& +4 \sum_{l=0}^{L-1} \sum_{l^{\prime} \neq l}\left|h_{l}\right|^{2}\left|h_{l^{\prime}}\right|^{2} M P_{s}^{2} \\
\operatorname{var}\left[C_{k}\right] & =4 \sum_{l=0}^{L-1}\left|h_{l}\right|^{2} M P_{s} \frac{N_{0}}{2}+M \frac{N_{0}^{2}}{2}
\end{aligned}
$$

The derivations of the BER formula give the following expression

$$
B E R=\frac{1}{2} E_{h_{l}}\left[\operatorname{erfc}\left(\sqrt{\frac{\sum_{l=0}^{L-1}\left|h_{l}\right|^{2} E_{b}}{2 N_{0} \Gamma}}\right)\right]
$$

with

$$
\Gamma=1+\frac{\sum_{l=0}^{L-1}\left|h_{l}\right|^{4} E_{b}}{\sum_{l=0}^{L-1}\left|h_{l}\right|^{2} M N_{0}}+\frac{M N_{0}}{2 \sum_{l=0}^{L-1}\left|h_{l}\right|^{2} E_{b}}+\frac{\sum_{l=0}^{L-1} \sum_{l^{\prime} \neq l}\left|h_{l}\right|^{2}\left|h_{l^{\prime}}\right|^{2} E_{b}}{\sum_{l=0}^{L-1}\left|h_{l}\right|^{2} M N_{0}}
$$

\section{Practical implementation issues}

Let us now consider some practical implementation issues on the structure of the transmitter and the receiver as presented in Figure 1. First of all, it has to be noted that in this paper we consider a digital implementation of the receiver, based on software defined radio (SDR) technology. Hence the received baseband signal is a digitised signal with a given oversampling rate. In a classical digital receiver, the received signal will be down converted to baseband using an in-phase quadrature (IQ)-demodulator. In this case, the baseband signal will be a complex signal modulated with a very low frequency component equal to the frequency shift between the received carrier and the local carrier. If the phase drift $\Delta \phi_{D}$ over the delay period $D$ between the two carriers due to this frequency offset is small, the useful signal will slightly degrade by a factor $\cos \left(\Delta \phi_{D}\right)$. In practice, the condition that the phase drift is very small over the delay period $D$ is not too hard to satisfy. For instance, for a chip rate of $2 \mathrm{Mchips} / \mathrm{s}$ and $D=2$ chips, a frequency offset of $10 \mathrm{kHz}$ between the received carrier and the local carrier, will degrade the useful signal only by a factor $\cos \left(\Delta \phi_{D}\right)=0.998$. As a consequence, we do not have to implement a frequency and phase recovery circuit in the receiver. 
A second practical consideration is on the delay $D$. In a digital implementation, the delay line implementation is straight forward using a shift register. The only concern could be the stability of the clocks yielding to slightly different delays in transmitter and receiver. To evaluate the influence of this difference between the two delays, some simulations were performed in Matlab. With a clock stability of $10^{-6}$ at both transmitter and receiver side, which is not too hard to meet, the simulation results revealed no significant degradation of the BER performance.

As a conclusion we can say that the structure of the DADS receiver can be kept very simple. First of all, there is no more need for spreading code alignment, as the reference signal is integrated in the received signal. Further, it is shown that under certain conditions there is no more need for a carrier recovery circuit.

The fact that the receiver structure is simple can also be considered as a drawback. Although DADS is a LPD modulation, it is very easy to demodulate the transmitted signal once it is detected, as the spreading code does not have to be known at the receiver side. The only parameter needed for the demodulation is the delay $D$, which is easy to extract from the captured signal by performing an autocorrelation. This motivates the use of fast frequency hopping to have a LPI modulation.

\section{THEORETICAL ANALYSIS OF THE BER PERFORMANCE OF THE DADS MODULATION SCHEME WITH FAST FREQUENCY HopPING IN FREQUenCy SElective RAYLEIGH CHANNELS}

Fast frequency hopping is defined as a hopping scheme in which the hop rate is higher than the symbol rate. In this paper, the frequency changes at a rate $K / M$ compared to the information rate $1 / M$, with $K$ the number of frequency hops necessary to span a data bit. Since the transmission of a data bit utilises more than one frequency hop, frequency diversity is obtained with fast frequency hopping signaling. For the DADS modulation scheme with an arbitrary PRBS, assuming that the delay $D$ is larger than the maximum delay spread $\tau_{\max }$, but significantly lower than the duration of a frequency hop $M / K$ $\left(\tau_{\max } \leq D \ll M / K\right)$, the BER becomes

$$
B E R=\frac{1}{2} E_{h_{l}^{k}}\left[\operatorname{erfc}\left(\sqrt{\frac{\frac{E_{b}}{K} \sum_{k=0}^{K-1} \sum_{l=0}^{L-1}\left|h_{l}^{k}\right|^{2}}{8 N_{0} \Gamma}}\right)\right]
$$

with $E_{h_{l}^{k}}[$.$] the expected value over the channel realisations$ and frequency hops, $h_{l}^{k}$ the complex-valued channel attenua- tion for the $l^{\text {th }}$ tap and the $k^{\text {th }}$ hop and

$$
\begin{gathered}
\Gamma=1+\frac{\frac{3 E_{b}}{K} \sum_{k=0}^{K-1} \sum_{l=0}^{L-1}\left|h_{l}^{k}\right|^{4}}{\frac{4 M N_{0}}{K} \sum_{k=0}^{K-1} \sum_{l=0}^{L-1}\left|h_{l}^{k}\right|^{2}}+\frac{M N_{0}}{\frac{2 E_{b}}{K} \sum_{k=0}^{K-1} \sum_{l=0}^{L-1}\left|h_{l}^{k}\right|^{2}} \\
+\frac{\frac{E_{b}}{K} \sum_{k=0}^{K-1} \sum_{l=0}^{L-1} \sum_{l^{\prime} \neq l}\left|h_{l}^{k}\right|^{2}\left|h_{l^{\prime}}^{k}\right|^{2}}{\frac{M N_{0}}{K} \sum_{k=0}^{K-1} \sum_{l=0}^{L-1}\left|h_{l}^{k}\right|^{2}}
\end{gathered}
$$

For the DADS modulation scheme with PRBS selection according to II-B, and assuming that the delay $D$ is larger than the maximum delay spread $\tau_{\max }$, but significantly lower than the length of a frequency hop $M / K\left(\tau_{\max } \leq D \ll M / K\right)$, the BER becomes

$$
B E R=\frac{1}{2} E_{h_{l}^{k}}\left[\operatorname{erfc}\left(\sqrt{\frac{\frac{E_{b}}{K} \sum_{k=0}^{K-1} \sum_{l=0}^{L-1}\left|h_{l}^{k}\right|^{2}}{2 N_{0} \Gamma}}\right)\right]
$$

with

$$
\begin{aligned}
& \Gamma=1+\frac{\frac{E_{b}}{K} \sum_{k=0}^{K-1} \sum_{l=0}^{L-1}\left|h_{l}^{k}\right|^{4}}{\frac{M N_{0}}{K} \sum_{k=0}^{K-1} \sum_{l=0}^{L-1}\left|h_{l}^{k}\right|^{2}}+\frac{M N_{0}}{\frac{2 E_{b}}{K} \sum_{k=0}^{K-1} \sum_{l=0}^{L-1}\left|h_{l}^{k}\right|^{2}} \\
& +\frac{\frac{E_{b}}{K} \sum_{k=0}^{K-1} \sum_{l=0}^{L-1} \sum_{l^{\prime} \neq l}^{L}\left|h_{l}^{k}\right|^{2}\left|h_{l^{\prime}}^{k}\right|^{2}}{\frac{M N_{0}}{K} \sum_{k=0}^{K-1} \sum_{l=0}^{L-1}\left|h_{l}^{k}\right|^{2}}
\end{aligned}
$$

\section{Simulation Results}

In order to evaluate the theoretical formulas given in Section II, we have simulated the baseband transmission chain given in Figure 1. Note that the performance results are presented for a spreading code $M=2048$ and delay $D=32$, but all the conclusions made for these specific values are also valid for any $M$ and $D$ assuming that $D$ is larger than the maximum delay spread $\tau_{\max }$, but significantly lower than the length of a spreading code $M$ or a frequency hop $M / K$.

\section{A. DADS in AWGN and frequency selective Rayleigh channels}

Figure 2 shows the theoretical and simulated BER performance of the DADS modulation scheme using arbitrary PRBS in AWGN and frequency selective Rayleigh channels for a spreading code $M=2048$, delay $D=32$, maximum delay spread $\tau_{\max }=16$, and various numbers of taps $L=$ $2,4,8,16$ which are zero-mean complex Gaussian variables independent and identically distributed (i.i.d.) with equal gains $1 / \sqrt{L}$. Simulations reveal that for BER values higher than $10^{-4}$, the BER curves for DADS modulation coincide for all frequency selective Rayleigh channels. Moreover, the more the number of taps $L$, the better the BER performance owing to the exploitation of the diversity order. Therefore, the DADS receiver can exploit the full diversity offered by the channel 


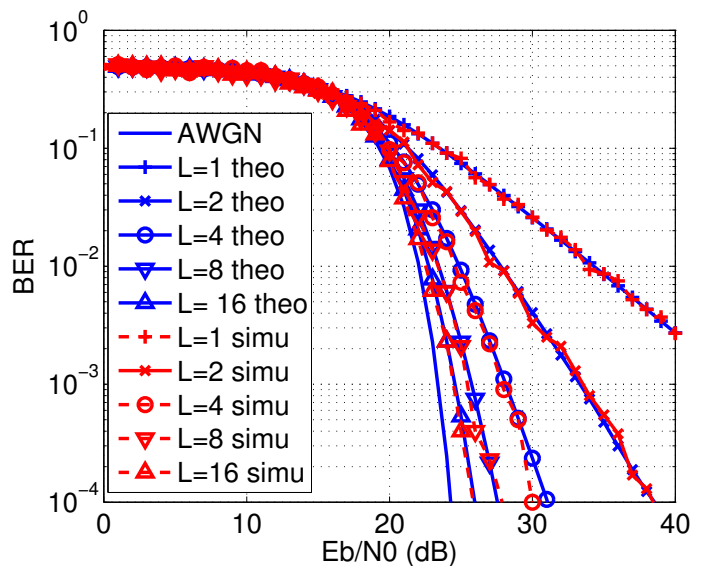

Fig. 2. Simulation results of the DADS modulation scheme using arbitrary PRBS in AWGN and frequency selective Rayleigh channels for $M=2048$ and $D=32$

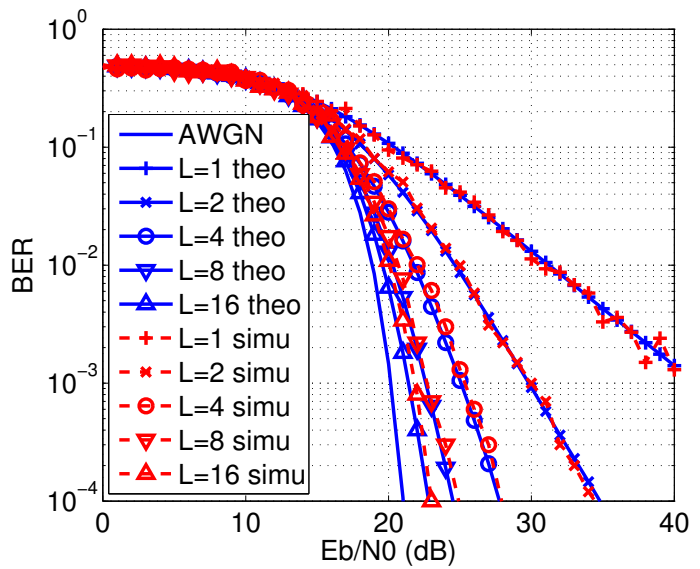

Fig. 3. Simulation results of the DADS modulation scheme using PRBS selection in AWGN and frequency selective Rayleigh channels for $M=2048$ and $D=32$

without adding complexity in the receiver, contrary to coherent DSSS modulations in which a large number of Rake fingers would be needed.

Figure 3 shows the theoretical and simulated BER performance of the DADS modulation scheme using PRBS selection in AWGN and frequency selective Rayleigh channels for a spreading code $M=2048$, delay $D=32$, maximum delay spread $\tau_{\max }=16$, and various numbers of taps $L=2,4$, 8,16 which are zero-mean complex Gaussian variables i.i.d. with equal gains $1 / \sqrt{L}$. To generate the PRBS satisfying (11), we choose a pseudo code of length $N=64$ chips satisfying $E\left[x_{i} x_{i-D}\right]=0$ which repeats itself to generate the spreading code for length $M$. The simulations show that the theoretical and simulated BER curves satisfying the criterion (11) coincide for frequency selective Rayleigh channels. A gain in performance of about $3 \mathrm{~dB}$ appears in frequency selective Rayleigh channel compared to arbitrary PRBS. Similar results are obtained for different values of $D$ as long as (11) is satisfied. Therefore, the DADS receiver with PRBS selection can also exploit the full diversity offered by the channel

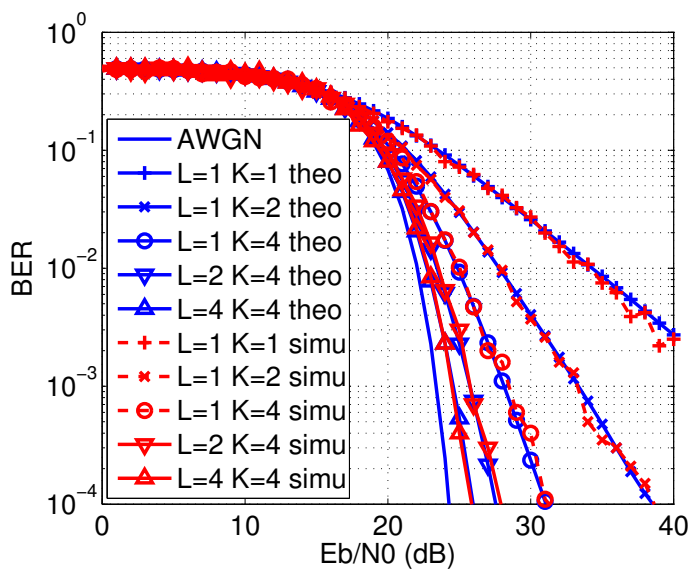

Fig. 4. Simulation results of the DADS modulation scheme using arbitrary PRBS with fast frequency hopping in AWGN and frequency selective Rayleigh channels for $M=2048$ and $D=32$

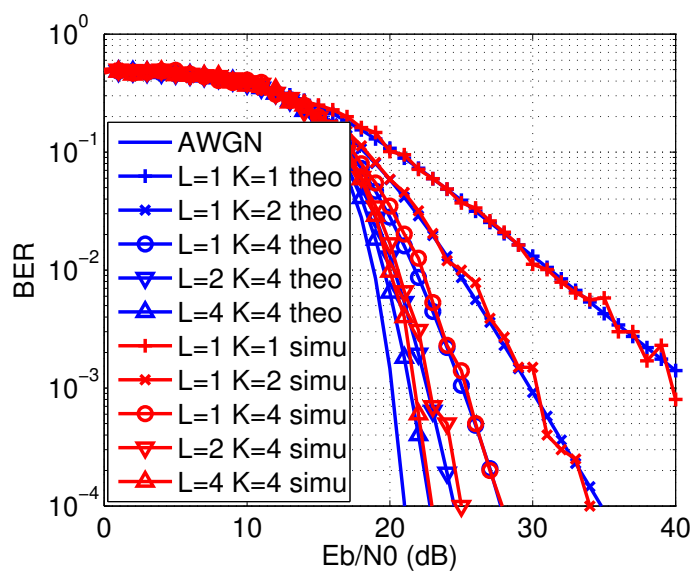

Fig. 5. Simulation results of the DADS modulation scheme using PRBS selection with fast frequency hopping in AWGN and frequency selective Rayleigh channels for $M=2048$ and $D=32$

without adding complexity in the receiver.

\section{B. DADS with Fast frequency hopping in $A W G N$ and fre- quency selective Rayleigh channels}

Figure 4 shows the theoretical and simulated BER performance of the DADS modulation scheme using arbitrary PRBS with fast frequency hopping in AWGN and frequency selective Rayleigh channels for a spreading code $M=2048$, delay $D=32$, maximum delay spread $\tau_{\max }=16$, various numbers of taps $L=2,4$ and various numbers of frequency hops $K=2$, 4 with $L$ i.i.d. zero-mean complex Gaussian taps having equal gains $1 / \sqrt{L}$ for each frequency hop. The BER curves coincide for all numbers of taps and numbers of frequency hops. Therefore, the addition of fast frequency hopping signaling exploits the full diversity offered by the channel while providing LPD/LPI to the modulation scheme.

Figure 5 shows the theoretical and simulated BER performance of the DADS modulation scheme using PRBS selection with fast frequency hopping in AWGN and frequency selective 
Rayleigh channels for a spreading code $M=2048$, delay $D=$ 32 , maximum delay spread $\tau_{\max }=16$, various numbers of taps $L=2,4$ and various numbers of frequency hops $K=$ 2, 4 with $L$ i.i.d. zero-mean complex Gaussian taps having equal gains $1 / \sqrt{L}$ for each frequency hop. The same PRBS as Figure 3 is used for the simulations. One can observe that the theoretical and simulated BER curves satisfying the criterion (11) coincide for all numbers of taps and numbers of frequency hops. The gain in performance of about $3 \mathrm{~dB}$ is still conserved with fast frequency hopping signaling, while exploiting the full diversity offered by the channel with LPD/LPI.

\section{CONCLUSION}

In DADS, a copy of the spreading code, delayed by $D$, is embedded in the transmitted signal. At the receiver, the signal is again delayed by $D$ and multiplied with the nondelayed signal for despreading, without the need for code synchronisation. This results in a very simple receiver structure, without loosing the benefits of a spread spectrum modulation, like e.g. the processing gain, LPD and interference resistance. Although DADS is a LPD modulation, it is very easy to demodulate the transmitted signal once it is detected, as the spreading code does not have to be known at the receiver side. The only parameter needed for the demodulation is the delay $D$, which is easy to extract from the captured signal by performing an autocorrelation. This motivates the use of fast frequency hopping to have a LPI modulation. In this paper, the performance of the DADS modulation scheme has been studied with fast frequency hopping in frequency selective Rayleigh channels. Theoretical analysis and simulation results have shown that the BER performance of DADS is improved drastically in frequency selective Rayleigh channels although keeping the same non-coherent receiver. Therefore, the DADS receiver can exploit the full diversity offered by the channel without adding complexity in the receiver, contrary to coherent DSSS modulations in which a large number of Rake fingers would be needed. Moreover, the BER performance of DADS is also enhanced with fast frequency hopping due to an increase in the diversity order of the frequency selective Rayleigh channels and provides LPD/LPI to the modulation scheme.

\section{REFERENCES}

[1] M. Sushchik, L. S. Tsimring, and A. R. Volkovskii, "Performance Analysis of Correlation-Based Communication Schemes Utilizing Chaos," IEEE Transactions on Circuits and SystemsI: Fundamental Theory and Application, vol. 47, no. 12, pp. 1684-1691, Dec. 2000.

[2] W. M. Tam, F. C. M. Lau, and C. K. Tse, "Generalized CorrelationDelay-Shift-Keying Scheme for Noncoherent Chaos-Based Communication Systems," IEEE Transactions on Circuits and Systems-I: Regular Papers, vol. 53, no. 3, pp. 712-721, Mar. 2006.

[3] B. Scheers and V. Le Nir, "A Modified Direct-Sequence Spread Spectrum Modulation Scheme for Burst Transmissions," Military Communications and Information Systems Conference (MCC'2010), Wroclaw, Poland, Sep. 2010.

[4] — " "Pseudo-Random Binary Sequence Selection for Delay and Add Direct Sequence Spread Spectrum Modulation Scheme," IEEE Communications Letters, vol. 14, no. 11, pp. 1002-1004, Nov. 2010.

[5] M. C. Jeruchim, "Techniques for estimating the Bit Error Rate in the simulation of Digital Communication Systems," IEEE Journal on Selected Areas in Communication, vol. SAC-2, no. 1, pp. 153-170, Jan. 1984.

[6] J. G. Proakis and M. Salehi, "Digital Communications, Fifth Edition," Mc Graw-Hill International Edition, New York, US, 2008. 